\title{
Electric-field-controlled spin reversal in a quantum dot with ferromagnetic contacts
}

\author{
J. R. HAUPTMANN*, J. PAASKE AND P. E. LINDELOF \\ The Niels Bohr Institute and The Nano-Science Center, University of Copenhagen, DK-2100 Copenhagen, Denmark \\ *e-mail: rahlf@fys.ku.dk
}

Published online: 30 March 2008; doi:10.1038/nphys931

Manipulation of the spin states of a quantum dot by purely electrical means is a highly desirable property of fundamental importance for the development of spintronic devices such as spin filters, spin transistors and single spin memories as well as for solid-state qubits ${ }^{1-6}$. An electrically gated quantum dot in the Coulomb blockade regime can be tuned to hold a single unpaired spin-1/2, which is routinely spin polarized by an applied magnetic field ${ }^{7}$. Using ferromagnetic electrodes, however, the quantum dot becomes spin polarized by the local exchange field $^{8-11}$. Here, we report on the experimental realization of this tunnelling-induced spin splitting in a carbon-nanotube quantum dot coupled to ferromagnetic nickel electrodes with a strong tunnel coupling ensuring a sizeable exchange field. As charge transport in this regime is dominated by the Kondo effect, we can use this sharp many-body resonance to read off the local spin polarization from the measured bias spectroscopy. We demonstrate that the exchange field can be compensated by an external magnetic field, thus restoring a zero-bias Kondo resonance, and we demonstrate that the exchange field itself, and hence the local spin polarization, can be tuned and reversed merely by tuning the gate voltage.

Since their discovery, carbon nanotubes (CNTs) have been intensively studied for their unique electrical properties. Their high Fermi velocity and low content of nuclear spins make them particularly well suited for spintronics applications using the transformation of spin information into electrical signals. Spin-valve effects in nanojunctions with a CNT spanning two ferromagnetic electrodes have already been observed ${ }^{12-15}$, and a strong gate dependence of the tunnel magnetoresistance has been demonstrated for CNT quantum dots in both the Coulomb blockade and the Fabry-Perot regime ${ }^{13}$.

In the intermediate coupling regime, odd-numbered quantum dots exhibit the Kondo effect, seen as a pronounced zero-bias conductance peak at temperatures below a characteristic Kondo temperature ${ }^{16-18}, T_{\mathrm{K}}$. This effect relies on the conduction electrons being able to flip the spin of the dot during successive cotunnelling events and is therefore expected to be sensitive to spin polarization of the electrodes. As pointed out by Martinek et al. ${ }^{9}$, quantum charge fluctuations, the electrons ability to tunnel on and off the dot, will renormalize the single-particle energy levels. In the case of ferromagnetic electrodes, this energy renormalization will be spin dependent and break the spin degeneracy on the dot, causing the spin states and thereby the zero-bias Kondo peak to split in two. This tunnelling-induced exchange-field splitting of the Kondo peak was observed by Patsupathy et al. ${ }^{8}$ in an ungated electromigrated $\mathrm{Ni}$ gap holding a $\mathrm{C}_{60}$ molecule. In contrast, the measurements here were made on a CNT quantum dot coupled to ferromagnetic Ni electrodes and, most importantly, with a back gate that enables us to tune the energy levels on the quantum dot. It is the presence of this back gate that now grants us experimental access to the interesting area of electrical spin control.

A singly occupied level residing just below the Fermi energy of the electrodes is strongly shifted by electrons tunnelling off and then back onto the dot, whereas a level deep below the Fermi energy (by almost the charging energy) is shifted by tunnelling of electrons onto and then off the dot. For spin-polarized electrodes with a difference in the density of spin-up and spin-down states, this implies a spin splitting of the dot level where sign and size depend on the applied gate voltage, that is, the position of the level below the Fermi energy. At the particle-hole symmetric point right between the empty and doubly occupied states, the spin degeneracy is expected to be intact ${ }^{19}$. In a material such as $\mathrm{Ni}$, however, we expect the band structure to be energy dependent and to have a Stoner splitting; this will break the particlehole symmetry and shift the spin degeneracy point away from the middle of the diamond ${ }^{10,11}$. Basically, all of the theoretical predictions for this pronounced gate dependence of the local spin states are experimentally verified by the transport measurements presented below.

Our sample geometry is shown in Fig. 1a with an external magnetic field applied in the direction of the Ni electrodes. We observe a clear even-odd effect with a zero-bias anomaly in every second Coulomb diamond, indicating a spin-1/2 Kondo effect with a typical Kondo temperature, $T_{\mathrm{K}} \sim 1 \mathrm{~K}$. Many of the observed Kondo anomalies showed a gate-dependent splitting and here we discuss two different devices for which this dependence is particularly clear; two other examples are shown in the Supplementary Information.

Magnetic force microscopy images of devices similar to the ones measured indicate that the CNT quantum dot is most likely coupled to one single domain in both the source and the drain electrodes. Applying a strong magnetic field serves partly to align the two contact domains and partly to provide a Zeeman splitting of the local spin. Figure 2 shows the conductance versus bias voltage and external field, measured for a gate voltage tuned to the middle of an odd-occupied Coulomb blockade valley in the Kondo regime. Device 1 (Fig. 2a,b) shows a simple linear behaviour in which the single-domain magnetization and hence the exchange field, $\mathbf{B}_{\mathrm{ex}}$, is aligned with the external field, $\mathbf{B}$. The exchange field can therefore be completely compensated and the zero-bias Kondo peak is seen to be restored at $B \sim \pm 1.12 \mathrm{~T}$, giving an indirect measure of the exchange field at this gate voltage. In device 2 


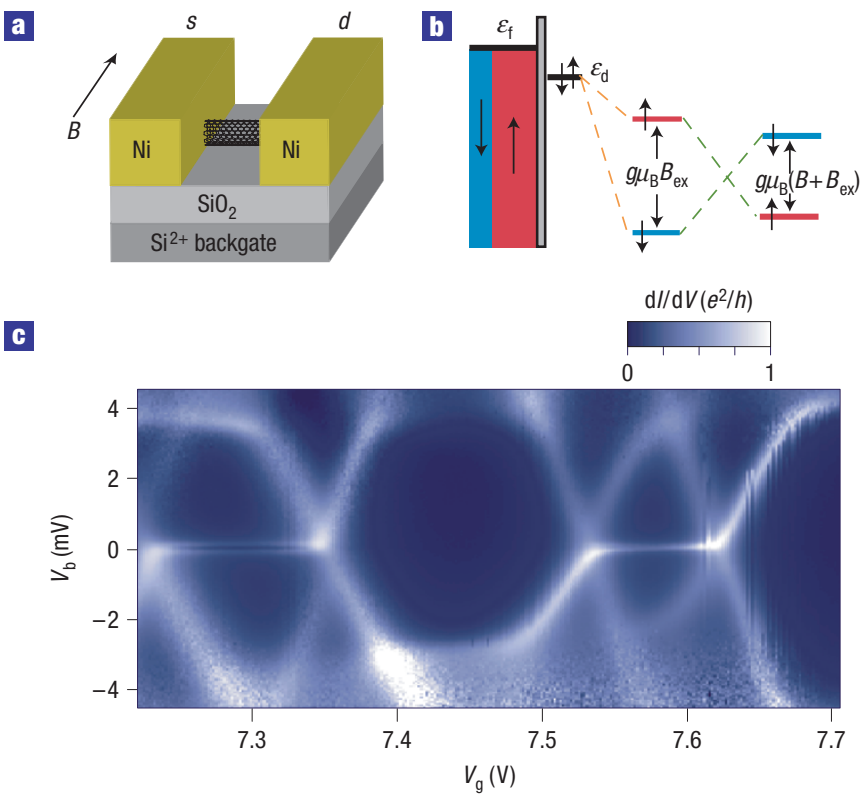

Figure 1 CNT quantum dot with magnetic contacts. a, Schematic view of the device comprising a segment of CNT on a Si/SiO${ }_{2}$ substrate, contacted by two $\mathrm{Ni}$ electrodes in a standard field-effect configuration. A magnetic field is applied along the electrodes in the plane of the substrate. $\mathbf{b}$, The local spin states are split partly by the tunnelling-induced exchange field $B_{\text {ex }}$ (in orange) that depends on gate voltage and partly by the external magnetic field $B$ (in green) resulting from the applied field $B$. c. Plot of the differential conductance $\mathrm{d} / \mathrm{d} V$ as a function of gate $V_{g}$ and bias voltage $V_{b}$ measured on device 2 . A series of four Coulomb diamonds can be observed with a pronounced zero-bias spin-1/2 Kondo peak in the two diamonds corresponding to odd-numbered occupation.

(Fig. 2c,d), on the other hand, the Kondo peak is never fully restored and the splitting merely reaches a minimum at $B \sim \pm 0.6 \mathrm{~T}$. This indicates that the exchange field lies at an angle to the external field, and fitting the $B$ dependence of the peak splitting by $e \Delta V=g \mu_{\mathrm{B}}\left|\mathbf{B}+\mathbf{B}_{\text {ex }}\right|$, we find this angle to be $\angle\left(\mathbf{B}, \mathbf{B}_{\mathrm{ex}}\right) \sim 25^{\circ}$ (see the Supplementary Information).

Figure 2 was recorded by sweeping the field from large negative to large positive values and a sudden decrease (increase) of the splitting is clearly visible at small negative (positive) fields. A similar switching was observed in ref. 8 and can be ascribed to a switching from the parallel to the antiparallel configuration of the contact domains driven by domain interactions (see the Supplementary Information). Figure $2 \mathrm{e}, \mathrm{f}$ shows the hysteretic behaviour around zero external field for devices 1 and 2, respectively. It can be observed that the switching, from the parallel to the antiparallel configuration, starts before the external magnetic field reaches zero (see the Supplementary Information) and that in both cases the conductance is enhanced in the antiparallel configuration where the tunnelling-induced exchange field is partially suppressed. The suppression depends on the symmetry of the coupling to the source and drain. Note also the hysteretic behaviour at higher magnetic fields, which we ascribe to small differences in the exact magnetization direction of the contact domains, that depends on the history of the external magnetic field. The difference in splitting of the Kondo peak in the parallel and antiparallel configurations can be used to estimate the coupling asymmetry. In terms of the conduction-electron spin polarization, $P=\left(v_{\mathrm{F} \uparrow}-v_{\mathrm{F} \downarrow}\right) / v_{\mathrm{F}}$, and the tunnel-broadening, $\Gamma_{\mathrm{s}(\mathrm{d})}=\pi v_{\mathrm{F}}\left|t_{\mathrm{s}(\mathrm{d})}\right|^{2}$, where $v_{\mathrm{F}}=v_{\mathrm{F} \uparrow}+v_{\mathrm{F} \downarrow}$ denotes the density of states in the electrodes and $t_{\mathrm{s}(\mathrm{d})}$ is the

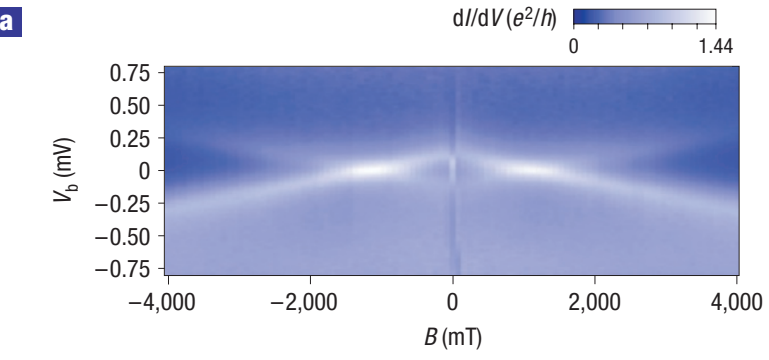

b
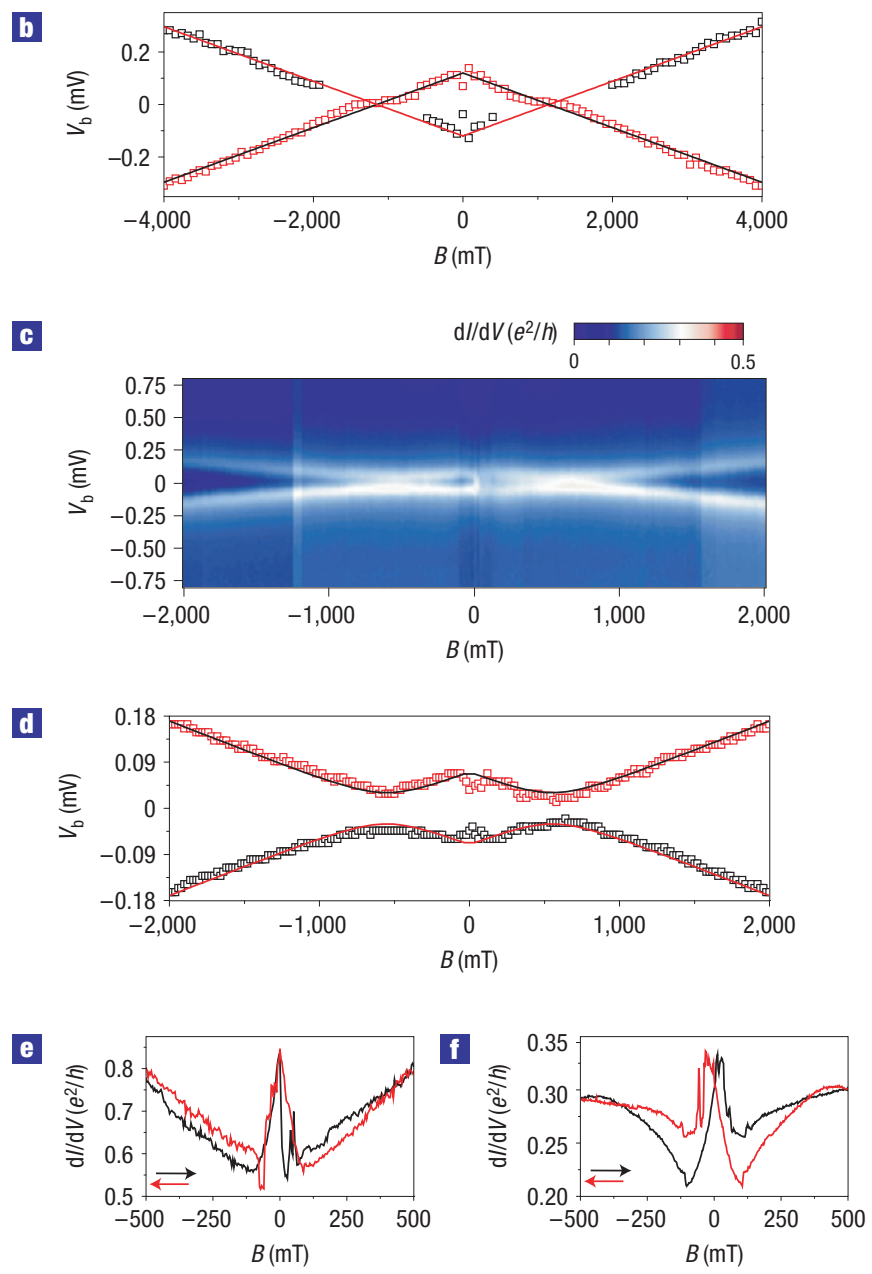

Figure 2 Kondo peak splitting as a function of applied magnetic field at fixed gate voltage. $a, d / / d V$ versus bias voltage and magnetic field measured on device 1 , sweeping from -4 to $4 \mathrm{~T}$. The plot is recorded in the middle of an odd-numbered spin-1/2 Kondo diamond. The observed splitting of the Kondo peak is due to the exchange field $B_{\text {ex }}$ and external field $B$. At $B \sim \pm 1.12 \mathrm{~T}$, the external field is compensating the exchange field and restoring the zero-bias Kondo peak. A sudden change in $\mathrm{d} l / \mathrm{d} V$ is observed at $B \sim-80 \mathrm{mT}$ due to a switching of one of the two contact domains. At $B \sim 80 \mathrm{mT}$, the other domain has flipped and the device is back in a parallel configuration. $\mathbf{b}$, Plot of the peak positions from $\mathbf{a}$, offset with $V_{b}=5 \mu \mathrm{V}$ to symmetrize the plot. The black and red lines are fits to the peak positions and have a slope of $\pm 1.04 \times 10^{-4} \mathrm{~V} \mathrm{~T}^{-1}$ corresponding to $g \sim 1$.8. c, Plot as in a, measured on device 2 , sweeping from -2 to $2 \mathrm{~T}$. In this device, the exchange field is never completely compensated, indicating a misalignment of the domain magnetization direction and the applied magnetic field, B. A domain switch causes a jump in the splitting at $B \lesssim|60| \mathrm{mT}$. d, Peak positions from $\mathbf{C}$, offset by $V_{b}=12 \mu \mathrm{V}$. The black and red lines are fits to the plots where the domain magnetization direction is at an angle of $25^{\circ}$ to the external field (see text). e,f, Plots of the spin-valve behaviour measured on devices 1 and 2 respectively. 


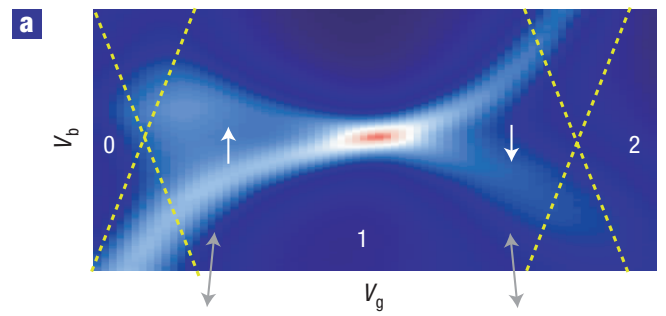

b

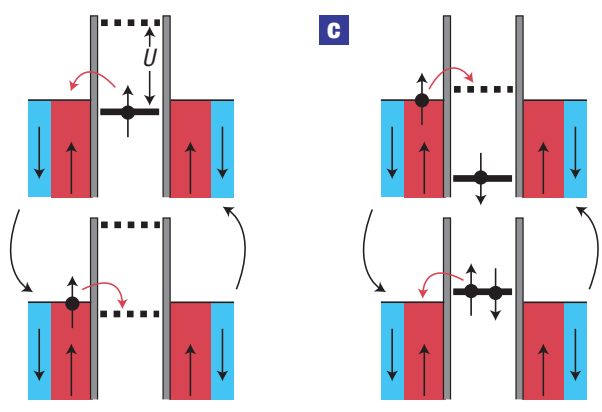

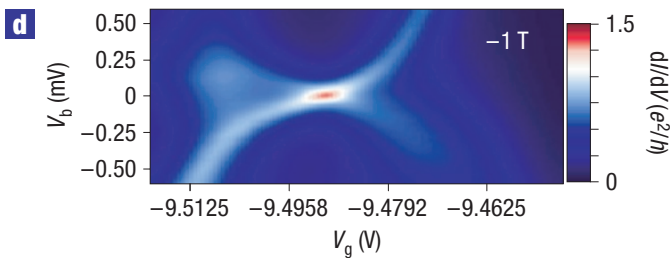

e

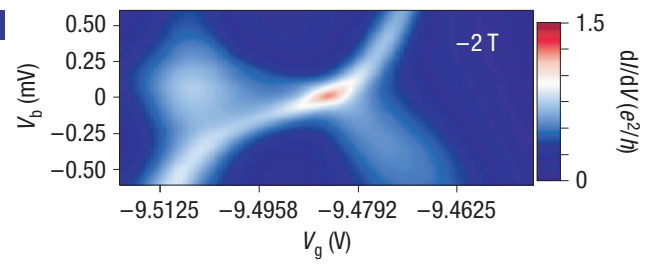

f
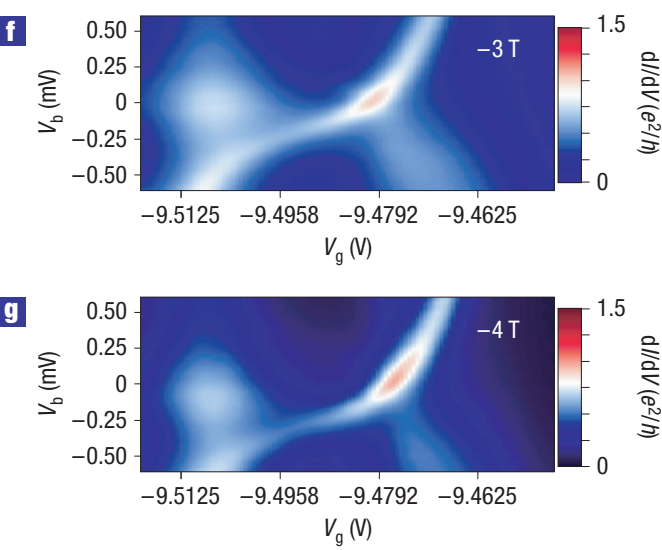

Figure 3 Gate dependence of the spin states probed by the Kondo effect. a, Plot from d, with yellow dashed lines added to indicate the diamond edges. Numbers indicate the number of electrons in one orbital state. Arrows indicates the local spin ground state which changes with the exchange field as the gate voltage is varied. These spin ground states on the dot are consistent with the observation in $\mathbf{d}-\mathbf{g}$, that the conductance peak (red spot) moves to the right as the field is increased. $\mathbf{b}, \mathbf{c}$, Illustration of the virtual tunnelling processes leading to a spin-up (down) ground state in the left (right) side of the dot; the solid lines are filled states and the dotted lines are empty states. Upper and lower diagrams correspond to ground and excited states. Red spin-up represents the majority $s$ electron band in the electrodes, hybridizing the most with the dot electrons. $\mathbf{d}-\mathbf{g}$, Plots of $d / / d V$ as a function of gate and bias voltage, measured on device 1 in different external magnetic fields. The Kondo peak is clearly seen to have a gate-dependent splitting. Full compensation in the middle of the diamond is obtained close to $B=-1 \mathrm{~T}$ as seen in $\mathbf{d}$.

tunnelling amplitude to the source (drain), it is expected ${ }^{8,20}$ that $g \mu_{\mathrm{B}} B_{\mathrm{ex}}^{(\mathrm{AP} / \mathrm{P})}=\Delta \varepsilon_{\mathrm{ex}}^{(\mathrm{AP} / \mathrm{P})}=a P\left(\Gamma_{\mathrm{s}} \mp \Gamma_{\mathrm{d}}\right)$. Comparing the exchange fields in the parallel and antiparallel configurations, for device 1 , we deduce that $\Gamma_{\mathrm{s}} / \Gamma_{\mathrm{d}} \sim 3$, which corresponds to a zero-bias Kondo peak height of $\left(2 e^{2} / h\right) 4 \Gamma_{\mathrm{s}} \Gamma_{\mathrm{d}} /\left(\Gamma_{\mathrm{s}}+\Gamma_{\mathrm{d}}\right)^{2}=1.5 e^{2} / h$ roughly consistent with the value of $1.4 e^{2} / h$, measured in the middle of the Coulomb blockade diamond for a fully compensating magnetic field near $B= \pm 1.12 \mathrm{~T}$.

In a Coulomb blockade diamond with an odd number of electrons, the left and right charge-degeneracy points correspond to emptying the dot or filling it by one extra electron, respectively. With a finite spin polarization in the electrodes, tunnelling of majority spins, spin-up for example, will be favoured by the higher density of states and a dot state of spin-up will therefore be shifted further down in energy than a spin-down state, as long as one is closer to the left-hand side of the diamond. Owing to the Pauli principle, the majority spins can only tunnel into the dot if the residing electron is in a spin-down state. It is therefore the spindown state that is lowered the most near the right-hand side of the diamond. This simple mechanism of level renormalization, shown in Fig. 3a-c, is encoded in the exchange field, given to good accuracy by

$$
\Delta \varepsilon_{\mathrm{ex}}\left(\varepsilon_{\mathrm{d}}\right)=e \Delta_{0}+(P \Gamma / \pi) \ln \left(\left|\varepsilon_{\mathrm{d}}\right| /\left|U+\varepsilon_{\mathrm{d}}\right|\right)
$$

to leading order in the tunnelling amplitude ${ }^{11}$ and assuming a constant density of states. $U$ is the charging energy and $\varepsilon_{\mathrm{d}}$ is the dot level position which is proportional to the gate voltage. Note the strong negative and positive logarithmic corrections for $\varepsilon_{\mathrm{d}}$ close to 0 or $-U$, respectively (corresponding to the left and right borders of the diamond). In the middle of the diamond, $\varepsilon_{\mathrm{d}}=-U / 2$, the exchange field is zero except for a constant term, $\Delta_{0}$, which we ascribe partially to a Stoner splitting between the spin-up and spin-down bands ${ }^{10,11}$, and partially to the spin splitting arising from spin-dependent interfacial phase shifts picked up by dot electrons on reflection on the ferromagnetic electrodes ${ }^{21}$. Note that possible stray fields from the magnetic contacts would also contribute with a gate-independent spin splitting (see the Supplementary Information).

Depending on the magnitude of $\Delta_{0}$, equation (1) predicts a change of the ground-state spin direction as the localized level is moved from $\varepsilon_{\mathrm{d}}=0$ to $\varepsilon_{\mathrm{d}}=-U$. Such a spin reversal can be observed in Fig. $3 \mathrm{~d}-\mathrm{g}$ and occurs at the conductance peak where the Kondo ridges cross and the spin states are degenerate. The movement of the conductance peak (red dot) as function of magnetic field, seen in Fig. 3d-g, confirms that the ground-state spin can indeed be reversed by changing the gate voltage. Furthermore, the direction of the motion shows that it is predominantly spin-up (that is, the spin along the 
a

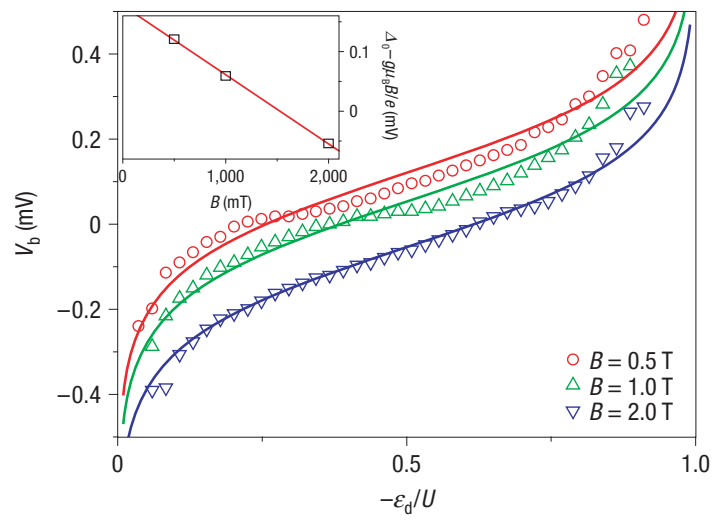

b

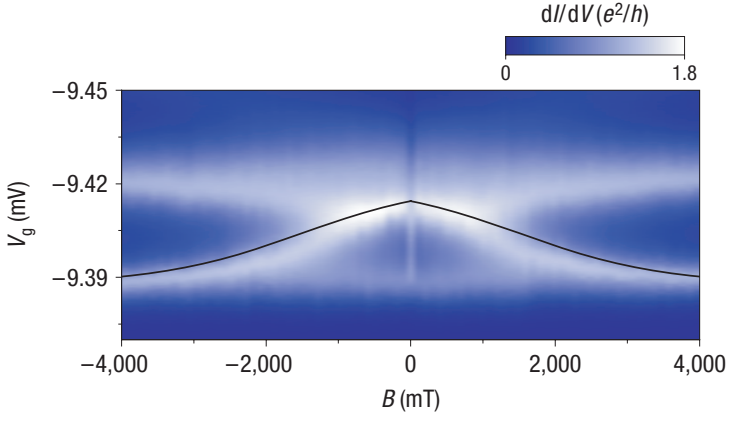

Figure $\mathbf{4}$ Gate dependence of the exchange field for different applied magnetic fields. a, Scatter plot of the dominant Kondo peak position, in bias voltage, as a function of level position $\varepsilon_{d}\left(\alpha-V_{g}\right)$ gate voltage for device 1. Peak positions are read off from plots similar to those in Fig. 3d-g (see the Supplementary Information). The different colours correspond to different magnetic fields. The gate axis has been displaced and normalized with the charging energy such that the dot is emptied at $\varepsilon_{d}=0$ and becomes doubly occupied at $\varepsilon_{d}=-U$. Solid lines are fits to the data, using $\left(\Delta \varepsilon_{\mathrm{ex}}-g \mu_{\mathrm{B}} B\right) / e$ defined by equation (1) and with $g=2$. All three curves are fitted by $P \Gamma / \pi=0.113 \mathrm{mV}$. Inset: A fit to the gate-independent term $\Delta_{0}-g \mu_{\mathrm{B}} B / e$ with $\Delta_{0}=0.18 \mathrm{mV} . \mathbf{b}, \mathrm{d} / / \mathrm{d} V$ as a function of gate voltage and magnetic field measured at zero bias voltage. The two nearly horizontal lines are Coulomb peaks and the white line moving from the lower to the upper peak as $B$ is lowered is a Kondo peak, thus mapping out where the spin states are degenerate. The black line is described by the function $\varepsilon_{d}=U /\left(\mathrm{e}^{\left(e \Delta_{0}-g \mu_{B} B\right) \pi / P \Gamma}+1\right)-c$, where $c$ is a constant determined by the first Coulomb peak and $U$ is the charging energy. $c$ and $U$ are read off from the plot, whereas the other constants are determined from the fits in a. Note that, like in Fig. 2, a domain switch is seen at $|B| \lesssim 80 \mathrm{mT}$.

external magnetic field) electrons that tunnel on and off the dot, giving a ground state with spin-up at the left-hand side of the diamond and spin-down at the right-hand side. This confirms earlier observations in conventional superconducting $\mathrm{Ni}$ tunnel junctions ${ }^{22,23}$ indicating that the tunnelling electrons in $\mathrm{Ni}$ have majority spin, that is positive polarization. Most likely, the more mobile $s$ electrons taking part in the tunnelling are spin polarized by their hybridization with the localized $d$ electrons $s^{24,25}$.

To substantiate the detailed gate-voltage dependence of the exchange field, we have extracted the peak positions from plots such as those in Fig. $3 \mathrm{~d}-\mathrm{g}$ and fitted them by $\left(\Delta \varepsilon_{\mathrm{ex}}\left(\varepsilon_{\mathrm{d}}\right)-g \mu_{\mathrm{B}} B\right) / e$ with $\Delta \varepsilon_{\text {ex }}$ given by equation (1). The result is shown in Fig. 4 a, where the inset shows $\Delta_{0}-g \mu_{\mathrm{B}} B / e$ as a function of $B$. The slope of the line corresponds to $g=2$ as expected for a CNT. Figure $4 \mathrm{~b}$ maps out the movement of the point where the spin states are degenerate as a function of magnetic field and gate voltage. The degeneracy point is seen as the white ridge moving between the two nearly horizontal white lines, corresponding to the Coulomb peaks. The plot has a gratifying resemblance to the theory plots shown in refs 10,11 . Inverting equation (1) and using the fitting parameters deduced from Fig. $4 a$, we arrive at the parameter-free fit seen as the black line in Fig. 4b.

\section{METHODS}

\section{EXPERIMENTAL}

The CNTs were grown by chemical vapour deposition on a $\mathrm{SiO}_{2}$ wafer with a highly doped Si back gate. The ferromagnetic electrodes were made of pure $\mathrm{Ni}$ in strips of thickness $\sim 60 \mathrm{~nm}$, width $\sim 300-1,000 \mathrm{~nm}$ and separated by $200-400 \mathrm{~nm}$. All measurements were made in a ${ }^{3} \mathrm{He} /{ }^{4} \mathrm{He}$ dilution refrigerator, with a base temperature of $30 \mathrm{mK}$, corresponding to a minimum electron temperature of $80 \mathrm{mK}$, and using a standard a.c. set-up with asymmetric bias.

\section{NOTE}

Note that the plots in Fig. 3d-g have been recorded before a switch in device 1, whereas all other measurements taken on device 1 were recorded after. We show these plots because they bring out most clearly the movement of the degeneracy point. The corresponding plots recorded after the gate switch are shown in the Supplementary Information and are qualitatively similar.

\section{Received 19 November 2007; accepted 29 February 2008; published 30 March 2008.}

References

1. Wolf, S. A. et al. Spintronics: A spin-based electronics vision for the future. Science 294, 1488-1495 (2001).

2. Awschalom, D. D. \& Flatté, M. E. Challenges for semiconductor spintronics. Nature Phys. 3, 153-159 (2007).

3. Ohno, H. et al. Electric-field control of ferromagnetism. Nature 408, 944-946 (2000).

4. Recher, P., Sukhorukov, E. V. \& Loss, D. Quantum dot as spin filter and spin memory. Phys. Rev. Lett. 85, 1962-1965 (2000).

5. Folk, J. A., Potok, R. M., Marcus, C. M. \& Umansky, V. A gate-controlled bidirectional spin filter using quantum coherence. Science 299, 679-682 (2003).

6. Elzerman, J. M. et al. Single-shot read-out of an individual electron spin in a quantum dot. Nature 430, 431-435 (2004).

7. Lindelof, P. E., Borggreen, J., Jensen, A., Nygård, J. \& Poulsen, P. R. Electron spin in single wall carbon nanotubes. Phys. Scr. T 102, 22-29 (2002).

8. Patsupathy, A. N. et al. The Kondo effect in the presence of ferromagnetism. Science 306, 86-89 (2004).

9. Martinek, J. et al. Kondo effect in quantum dots coupled to ferromagnetic leads. Phys. Rev. Lett. 91, 127203 (2003).

10. Martinek, J. et al. Gate-controlled spin splitting in quantum dots with ferromagnetic leads in the Kondo regime. Phys. Rev. B 72, 121302 (2005).

11. Sindel, M. et al. Kondo quantum dot coupled to ferromagnetic leads: Numerical renormalization group study. Phys. Rev. B 76, 45321 (2007).

12. Tsukagoshi, K., Alphenaar, B. W. \& Ago, H. Coherent transport of electron spin in a ferromagnetically contacted carbon nanotube. Nature 401, 572-574 (1999).

13. Sahoo, S. et al. Electric field control of spin transport. Nature Phys. 1, 99-102 (2005).

14. Jensen, A., Hauptmann, J. R., Nygård, J. \& Lindelof, P. E. Magnetoresistance in ferromagnetically contacted single-wall carbon nanotubes. Phys. Rev. B 72, 35419 (2005).

15. Hueso, L. E. et al. Transformation of spin information into large electrical signals using carbon nanotubes. Nature 445, 410-413 (2007).

16. Glazman, L. \& Raikh, M. Resonant Kondo transparency of a barrier with quasilocal impurity states. JETP Lett. 47, 452-455 (1988).

17. Goldhaber-Gordon, D. et al. Kondo effect in a single-electron transistor. Nature 391, 156-159 (1998).

18. Nygård, J., Cobden, D. H. \& Lindelof, P. E. Kondo physics in carbon nanotubes. Nature 408, 342-346 (2000)

19. Choi, M. S., Sanchez, D. \& Lopez, R. Kondo effect in a quantum dot coupled to ferromagnetic leads: A numerical renormalization group analysis. Phys. Rev. Lett. 92, 56601 (2004).

20. Utsumi, Y., Martinek, J., Schön, G., Imamura, H. \& Maekawa, S. Nonequilibrium Kondo effect in a quantum dot coupled to ferromagnetic leads. Phys. Rev. B 71, 245116 (2005).

21. Cottet, A., Kontos, T., Belzig, W., Scönenberger, C. \& Bruder, C. Controlling spin in an interferometer with spin-active interfaces. Europhys. Lett. 74, 320-326 (2006).

22. Tedrow, P. M. \& Meservey, R. Spin-dependent tunneling into ferromagnetic nickel. Phys. Rev. Lett. 26, 192-195 (1971).

23. Kim, T. H. \& Moodera, J. S. Large spin polarization in epitaxial and polycrystalline Ni films. Phys. Rev. B 69, 20403 (2004).

24. Mazin, I. I. How to define and calculate the degree of spin polarization in ferromagnets. Phys. Rev. Lett. 83, 1427-1430 (1999).

25. Tsymbal, E. Y. \& Pettifor, D. G. Modelling of spin-polarized electron tunnelling from 3d ferromagnets. J. Phys. Condens. Matter 9, L411-L417 (1997).

\section{Acknowledgements}

We thank L. Borda, R. Gunnarsson, K. Flensberg, P. Hedegård and C. M. Marcus for discussions. For financial support, we thank the Danish Agency for Science Technology and Innovation (FTP 57730, FNU 272-05-0338) and EU projects SECOQC and CARDEQ.

Correspondence and requests for materials should be addressed to J.R.H.

Supplementary Information accompanies this paper on www.nature.com/naturephysics. 